\title{
SCARABAEIDAE (COLEOPTERA) NA MATA ATLÂNTICA: SISTEMAS AGROFLORESTAIS SÃO PERMEÁ VEIS A BESOUROS DE FLORESTA?
}

Jacirleide Lima Maciel ${ }^{1}$, Priscila Paixão Lopes $^{2}$

1.Bolsista PROBIC/UEFS, graduanda em Ciências Biológicas, Universidade Estadual de Feira de Santana, e-mail: jacy_bio@hotmail.com

2.Orientadora, Departamento de Ciências Biológicas, Universidade Estadual de Feira de Santana, e-mail: pplc29@gmail.com

Palavras-chave: ecologia de comunidades; diversidade; rola-bosta; semiárido

\section{INTRODUÇÃO}

A mata atlântica é uma das formações mais intensamente destruídas do Brasil, restando menos de 5\% de sua área original dispersos em fragmentos em níveis variados de isolamento (TABARELLI et al. 2005). As florestas tropicais são conhecidas por sua alta biodiversidade, contudo, perturbações antropogênicas modificam a paisagem e a estrutura da vegetação florestal, afetando a biodiversidade. As condições ambientais em uma floresta são amplamente diferentes das de uma área aberta. Portanto, a destruição dos ambientes naturais para a criação de áreas de pastagem ou de monoculturas provoca alterações nos fatores abióticos, podendo ocasionar uma mudança na composição e na estrutura das comunidades, já que as espécies adaptadas às condições florestais não se adaptam às novas condições ambientais (MEDRI \& LOPES 2001). Isso é registrado para muitos grupos, inclusive os Scarabaeinae (NICHOLS et al. 2007). A perda contínua de florestas tropicais intactas aumenta a importância potencial de Sistemas Agroflorestais (SAF) para a conservação dos artrópodes tropicais. Sistemas Agroflorestais sombreados ainda podem suportar alto nível de biodiversidade (BOS et al. 2007).

Os Scarabaeidae, conhecidos popularmente como rola-bostas, são detritívoros que usam majoritariamente excrementos, e retiram porções de recurso, levando-as até o local de alimentação ou reprodução (HALFFTER \& MATTHEWS 1966). De um modo geral se alimentam de excrementos de mamíferos, comunidade esta que responde igualmente à formação vegetal. Os Scarabaeinae coprófagos apresentam adaptações à composição, textura e umidade típicas de cada espécie de vertebrado (MARTIN \& LOBO 1993; MORELLI \& GONZALEZ-VAINER 1997). Os besouros de áreas abertas tenderão, portanto, a utilizar recursos que sáo abundantes nesse tipo de ambiente, ou seja, fezes de grandes herbívoros, enquanto que se espera que os besouros de áreas florestais estejam mais adaptados a utilizar as fezes de vertebrados com menor teor de fibras vegetais, e potencialmente provenientes de dietas onívoras (HALFFTER \& MATTHEWS 1966). Desta forma, podemos identificar os organismos que vivem predominantemente em cada tipo de ambiente através dos recursos que utilizam.

Com isso buscaremos ver se em áreas de SAF adjacentes a fragmentos de mata atlântica existe alguma troca de espécies entre as comunidades típicas de cada ambiente e o grau de penetração de espécies de Mata Atlântica em direção ao ambiente de SAF e vice versa. O trabalho tem, portanto, aplicabilidade direta em estudos de conservação e planejamento de corredores ecológicos através de sistemas agroflorestais.

\section{METODOLOGIA}

O estudo foi realizado na APA do Pratigi, no Baixo Sul da Bahia, que engloba os municípios de Ituberá, Nilo Peçanha, Igrapiúna, Piraí do Norte e Ibirapitanga. A amostragem das comunidades de Scarabaeinae foi realizada em seis fragmentos florestais do ecopolo I, com áreas variadas (Adeilton $=344$, Waldemar $=342$, André $=190$, Coco $=158, B r a n c o=17$ e Jairo=15,9ha), e SAF adjacentes a estes (fragmentos identificados a partir dos proprietários dos SAF). Todos os SAF têm instalação recente ( $<3$ anos) e cerca de 1 ha, com exceção do SAF de propriedade do Sr. Antônio de Souza (Coco), que tem 2,5ha e mais de 10 anos. Em 
cada área (SAF e fragmentos adjacentes) foram colocados seis pontos amostrais, distantes $50 \mathrm{~m}$ um do outro, sendo os pontos dos fragmentos $100 \mathrm{~m}$ para dentro da mata para evitar o efeito da borda. Cada ponto foi composto por duas armadilhas de queda sendo cada uma iscada com um tipo de fezes (bovinas e humanas). As armadilhas foram compostas por frascos plásticos com $15 \mathrm{~cm}$ de abertura e $10 \mathrm{~cm}$ de profundidade, enterradas até que a abertura estivesse à altura do solo. Na parte central de cada frasco foi posicionado um pote de $30 \mathrm{ml}$ de isca; no fundo do pote foram colocados cerca de $200 \mathrm{ml}$ de uma solução de água, detergente e sal. As armadilhas ficaram expostas por 48h, quando os espécimes foram coletados, conservados em álcool $70 \%$ e depois levados ao Laboratório de Entomologia da UEFS (LENT) para triagem, montagem e identificação. Todos os exemplares foram tombados na coleção entomológica do Museu de Zoologia da UEFS (MZFS). A abertura do dossel foi medida através de fotografias digitais com lente olho de peixe (hemisférica), e analisadas com o programa GLA v 2.0.

Os SAF e fragmentos florestais foram comparados em sua composição (NMDS com métrica de Bray-Curtis, seguido de ANOSIM em caso de stress < 0,2), resposta aos dois tipos de isca (como indicação de adaptação a recursos mais comuns em áreas abertas), além de correlacionar abundância e riqueza com a área dos fragmentos e abertura do dossel (fragmentos e SAF).

\section{RESULTADOS E DISCUSSÃO}

Os SAF amostrados são adjacentes aos fragmentos de mata, apresentando em sua estrutura diversos tipos de plantios (banana, cacau, cupuaçu, etc.) que são utilizados como opção viável de interesse de recuperação de áreas degradadas. O fragmento de propriedade de Adeilton Pereira de Jesus foi o que apresentou maior abundância e riqueza de espécies e indivíduos, pois fragmentos maiores tem a capacidade de abrigar uma maior quantidade de indivíduos e também de recursos alimentares disponíveis para os besouros ali encontrados.

Durante a realização do projeto foram coletados 1061 indivíduos de 27 espécies. Dentre as espécies coletadas as mais abundantes foram Canthonella silphoides Harold 1867 (544 inds.) sendo 539 desses indivíduos encontrados nos fragmentos de Mata Atlântica e apenas cinco em fragmentos de SAF, e o Dichotomius (L.) gr. sericeus sp.nov. onde 205 foram encontrados em mata e três em SAF. Das 27 espécies coletadas nesses fragmentos apenas duas delas foram encontradas exclusivamente em fragmentos de SAF (Canthon $(C$.) prox. septenmaculatus e Uroxys sp1), oito espécies foram coletadas tanto em fragmentos de mata quanto em fragmentos de SAF (C. silphoides, Dichotomius (L.) gr. sericeus, D. (D.) mormon (Ljungh 1799), D. (D.) depresicollis Harold, 1867, Eurysternus nigrovirens Génier 2009, E. cyanescens Balthasar 1939, Onthophagus buculus Mannerhein 1829, O. haematopus Harold 1875), e 17 espécies encontradas exclusivamente em ambiente de Mata Atlântica.

A composição foi considerada similar entre os fragmentos e entre SAF (Figura 1), mas fragmentos e SAF foram dissimilares (ANOSIM, $\mathrm{p}=0,006$ ). A estrutura do dossel dos SAF é significativamente mais aberta que a estrutura do dossel dos fragmentos de mata (ANOVA: $\mathrm{F}(11,66)=34,708, \mathrm{p}=0,0000)$. Não há diferenças entre as matas e entre os SAF. Ou seja, o padrão de composição repetiu o padrão de abertura do dossel.

Conforme o esperado, foi encontrada uma maior riqueza e abundância de espécies e indivíduos de Scarabaeidae registradas em fragmentos de Mata Atlântica em comparação com os SAF (Tabela 1). Isso pode ser explicado tanto devido ao efeito de área, que foi significativa (comparando apenas os fragmentos de mata) $\left(r_{=} 0,6772 ; p<0,001\right)$, quanto pelo menor nível de abertura do dossel nos fragmentos florestais.

As fezes humanas atraíram mais indivíduos que fezes bovinas tanto em matas (FH: 993 ind.; FB: 14 ind.) quanto nos SAF (FH: 47 ind.; FB: 1 ind.). No que se relaciona à atratividade das iscas, na mata atlântica foi confirmada a preferência pelas fezes humanas, 
mas diferente do esperado, as fezes bovinas não foram mais atrativas nos SAF. Um dos motivos que pode ter gerado uma maior atratividade de Scarabaeidae pelas fezes humanas é pela similaridade com as fezes de onívoros que possuem mais componentes voláteis em relação ás fezes dos herbívoros. Como as fezes bovinas são mais frequentes em ambientes abertos como é a estrutura do SAF, poderiam atrair espécies de besouros que não são de interior de mata, mas a restrição de recursos para vertebrados no SAF pode ser suficiente para que os coprófagos generalistas (em termos da origem das fezes) utilizem o que estiver mais disponível e atrativo.

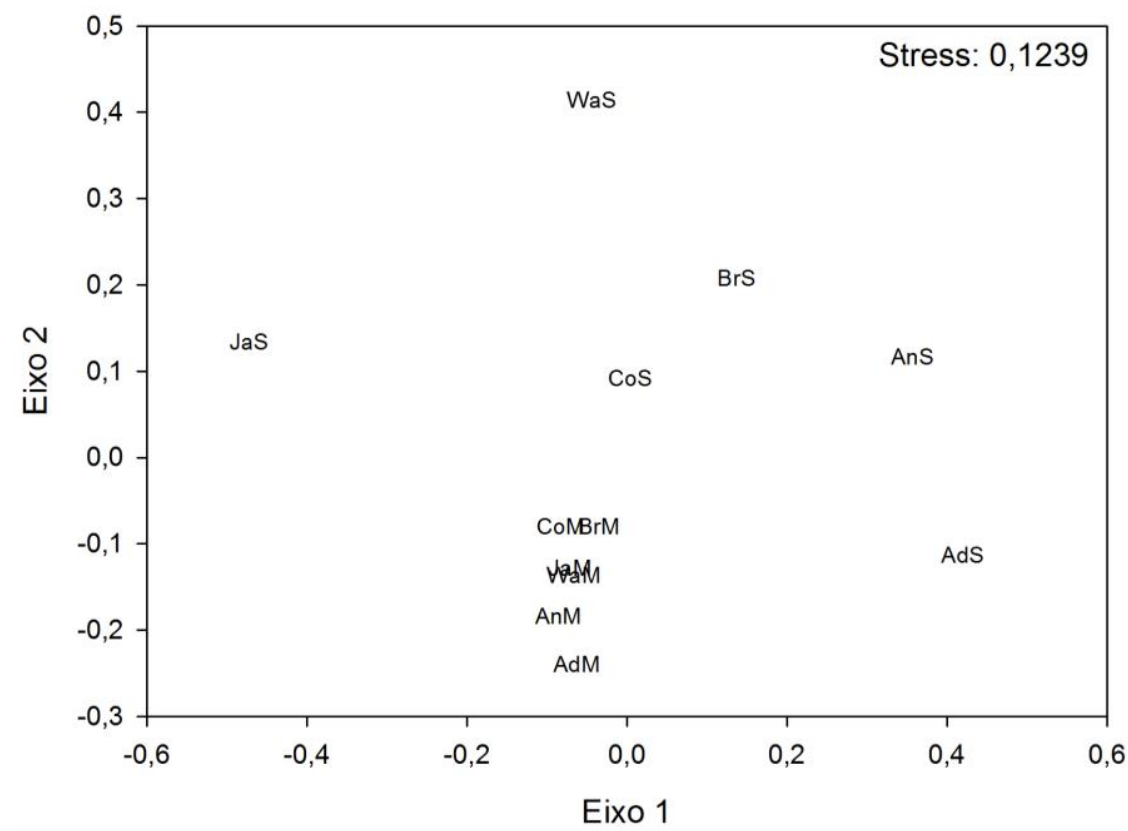

Figura 1. NMDS de similaridade de áreas $\mathrm{x}$ ambientes, métrica de Bray-Curtis. Composição dos códigos: $\mathrm{Ad}=$ Adeilton, $\mathrm{An}=\mathrm{André} ; \mathrm{Br}=\mathrm{Branco} ; \mathrm{Co}=\mathrm{Coco}$; Já=Jairo; Wa=Waldemar. $\mathrm{M}=\mathrm{Mata}$; $\mathrm{S}=\mathrm{SAF}$.

Tabela 1. Distribuição de riqueza e abundância de Scarabaeinae entre os locais e ambientes analisados na APA do Pratigi, e métricas dos ambientes (área e percentual de abertura do dossel). Fragmentos identificados a partir dos nomes dos proprietários dos SAF adjacentes aos fragmentos.

\begin{tabular}{cccccc}
\hline Local & Ambiente & N & S & Área (ha) & $\begin{array}{c}\text { Ab. Dossel } \\
(\%)\end{array}$ \\
\hline \multirow{2}{*}{ Adeilton } & MATA & 427 & 20 & 344 & 27,71 \\
& SAF & 12 & 4 & 1 & 72,56 \\
\multirow{2}{*}{ André } & MATA & 218 & 9 & 190 & 22,25 \\
& SAF & 2 & 2 & 1 & 60,46 \\
\multirow{2}{*}{ Branco } & MATA & 59 & 8 & 17 & 30,32 \\
& SAF & 6 & 4 & 1 & 75,20 \\
\hline \multirow{2}{*}{ Coco } & MATA & 67 & 9 & 158 & 23,47 \\
& SAF & 25 & 7 & 2,5 & 49,11 \\
\multirow{2}{*}{ Jairo } & MATA & 90 & 8 & 15,9 & 21,56 \\
\hline \multirow{2}{*}{ Waldemar } & SAF & 1 & 1 & 1 & 55,95 \\
\hline Total & MATA & 153 & 12 & 342 & 27,90 \\
\hline
\end{tabular}


Os SAF apresentaram um número muito baixo de espécies e indivíduos, como era esperado, sendo isso explicado por apresentarem uma estrutura de dossel significativamente mais aberta. As florestas tropicais são conhecidas por sua alta biodiversidade (TABARELLI et al. 2005), contudo as perturbações antropogênicas que muitos ambientes sofrem modificam a paisagem e a estrutura da vegetação, afetando consequentemente a biodiversidade do ambiente. O padrão de formação dos SAF mostra que uma vegetação mais aberta que a Mata Atlântica possa não permitir uma alta biodiversidade de besouros, possivelmente em função de uma menor disponibilidade e quantidade de recursos, que rapidamente se ressecam.

A composição diferente de fragmentos florestais e SAF indica uma não utilização dos SAF por organismos florestais em função da dissimilaridade estrutural da vegetação. A diferença pode ser explicada devido à maioria dessas áreas estarem em formação recente, tendo ainda árvores e vários tipos de plantios ainda em desenvolvimento, ou seja, tendo estrutura pouco desenvolvida. Mas a similaridade foi maior entre os fragmentos florestais e o SAF do Sr. Coco, que é maior, mais antigo e tem dossel mais fechado que os demais SAF. Isso sugere que à medida que a vegetação arbórea do SAF cresce e começa a formar uma copa, poderia passar a se tornar viável para o trânsito de insetos de florestas.

\section{CONSIDERAÇÕES FINAIS}

Confirmamos que a estrutura do dossel influencia na conservação e manutenção no número de espécimes dos Scarabaeidae, bem como o tamanho do fragmento. Também foi possível perceber que as matas apresentam menor variação de riqueza e abundância em seus fragmentos, ou seja, as comunidades de besouros permanecem mais estáveis nestes ambientes. As áreas de Mata Atlântica proporcionam uma maior disponibilidade de recursos, enquanto que as áreas de SAF, que por serem áreas mais abertas e em processo de formação não oferece proteção e nem recursos alimentares suficientes para os Scarabaeinae. Mas etapas posteriores de formação de SAF, com árvores frutíferas, bem como as madeiras nativas em sua altura máxima, podem efetivamente simular matas, o que deve ser mais adequadamente determinado com SAF mais antigos e de maior área.

\section{REFERÊNCIAS}

BOS, M.M. et al. 2007. Insect diversity responses to forest conversion and agroforestry management. In: Tscharntke, T. et al. (eds), The stability of tropical rainforest margins, linking ecological, economic and social constraints of land use and conservation, Springer Berlin, pp 279-296.

HALFFTER, G.; MATTHEWS, E.G. 1966. The natural history of dung beetles of the subfamily Scarabaeinae (Coleoptera, Scarabaeidae). Folia Entom. Mex. 12-14: 1-312.

MARTIN, P. F.; LOBO, J. M. 1993. Altitudinal distribution pattern of copro-necrophage Scarabaeidae (Coleoptera) in Veracruz, México. Coleopt. Bull. 47: 321-334.

MATAVELLI, R.A.; LOUZADA, J.N.C. 2008. Invasão de áreas de savana intra-amazônicas por Digitonthophagus gazella (Fabricius, 1787) (Insecta: Coleoptera: Scarabaeidae). Acta Amazonica 38(1):153-158.

MEDRI, I.M.; LOPES, J. 2001. Scarabaeidae (Coleoptera) do Parque Estadual da Mata dos Godoy e de área de pastagem, no norte do Paraná-Brasil. Rev. Bras. Zool. 18 (Supl. 1): 135141.

MORELLI, E.; GONZALEZ-VAINER, P. 1997. Dung beetles (Coleoptera: Scarabaeidae) inhabiting bovine and ovine dropping in Uruguayan prairies. Coleopt. Bull. 51: 197.

NICHOLS, E. et al. 2007. Global dung beetle response to tropical forest modification and fragmentation: A quantitative literature review and meta-analysis. Biol. Conserv., 137:1-19.

TABARELLI, M. et al. 2005. Desafios e oportunidades para a conservação da biodiversidade na Mata Atlântica brasileira. Megadiversidade, 1:132-138. 